\title{
Fentanyl Citrate Buccal Tablet
}

National Cancer Institute

\section{Source}

National Cancer Institute. Fentanyl Citrate Buccal Tablet. NCI Thesaurus. Code C83012.

A tablet formulation containing the citrate salt of the synthetic anilidopiperidine opiate fentanyl with analgesic activity. Upon contact with the buccal mucosa, fentanyl citrate buccal tablet rapidly releases fentanyl which is quickly absorbed into the systemic circulation. Fentanyl selectively binds to and activates mu-opioid receptors in the central nervous system (CNS), mimicking the effects of endogenous opioids. 